\title{
Joint Intensity Inhomogeneity Correction for Whole-Body MR Data
}

\author{
Oleh Dzyubachyk ${ }^{1}$, Rob J. van der Geest ${ }^{1}$, Marius Staring ${ }^{1}$, Peter Börnert ${ }^{1,2}$, \\ Monique Reijnierse $^{1}$, Johan L. Bloem ${ }^{1}$, and Boudewijn P.F. Lelieveldt ${ }^{1,3}$ \\ 1 Department of Radiology, Leiden University Medical Center, \\ Leiden, The Netherlands \\ 2 Philips Research Laboratories, Hamburg, Germany \\ 3 Intelligent Systems Department, Delft University of Technology, \\ Delft, The Netherlands
}

\begin{abstract}
Whole-body MR receives increasing interest as potential alternative to many conventional diagnostic methods. Typical whole-body MR scans contain multiple data channels and are acquired in a multistation manner. Quantification of such data typically requires correction of two types of artefacts: different intensity scaling on each acquired image stack, and intensity inhomogeneity (bias) within each stack. In this work, we present an all-in-one method that is able to correct for both mentioned types of acquisition artefacts. The most important properties of our method are: 1) All the processing is performed jointly on all available data channels, which is necessary for preserving the relation between them, and 2) It allows easy incorporation of additional knowledge for estimation of the bias field. Performed validation on two types of whole-body MR data confirmed superior performance of our approach in comparison with state-of-the-art bias removal methods.
\end{abstract}

Keywords: Whole-body MRI, multi-spectral MRI, multi-station acquisition, intensity inhomogeneity correction, intensity standardization.

\section{Introduction}

Whole-body (WB) MR has become a subject of intensive research for having a large potential in being an alternative to other imaging modalities with high anatomical detail. Nowadays, it has found clinical application in, among others, oncology, e.g. in assessment of multiple myeloma (MM), or cardiology, e.g. in obesity studies [1].

WB MR acquisitions are typically performed in multiple stages [2, a restriction imposed by the limited field of view of the MR scanner. For further processing, all the separately acquired image stacks need to be combined into a single volume. Such reconstructed volumes typically exhibit two types of irregularity:

\footnotetext{
* This research was supported by the Dutch Technology Foundation STW (Stichting
}

Technische Wetenschappen) via grant 10894. 
intensity inhomogeneity (bias) within each stack and a different dynamic intensity range for each station [3. Both inhomogeneities cause significant problems for further image processing, thus robust removal of these artifacts would be highly desired.

A number of methods has been developed during last years for correction of both types of intensity inhomogeneity. An extensive review of the bias correction methods can be found in [4]. For dealing with the second type of intensity inhomogeneity, several specialized methods have been developed [3].

Here we present a novel all-in-one method for volume reconstruction from multi-station multi-spectral WB MR data. The developed method performs correction of both types of intensity inhomogeneity. The main methodological contribution of this work is two-fold: 1) We extend the Coherent Local Intensity Clustering (CLIC) framework [5] to the multi-spectral data case with joint estimation of the bias field for all data channels (Section 2.2), that allows preserving relation between them; and 2) Two novel optimization constraints (Section 2.4) were added to the model for improved estimation of the bias field. The following section describes our method and its novel parts in full detail.

\section{Method}

Our method is based on the CLIC framework, which was extended in several parts to cope with the complexity of our data. More precisely, the following modifications were developed in comparison with the original method:

- All the processing is performed jointly on all available data channels. This is important for preserving correspondence between these channels.

- We consider the bias field $b$ to be additive, which is achieved by applying the logarithmic transformation to the general MR acquisition model

$$
I=J+b+n_{\text {bio }},
$$

where $I$ and $J$ are logarithms of the measured and the true signal correspondingly, and $n_{\text {bio }}$ is the "biological noise". Additive bias model is more appropriate when the tissue-related bias is more prominent than the hardware-related one, and drastically simplifies solving the constrained optimization model.

- The possibilistic clustering [6] is used instead of the probabilistic one.

- The scaled Mahalanobis distance [6] is used instead of the Euclidean one.

- The centers $\mathbf{c}_{i}$ and the fuzzy covariance matrices $\Sigma_{i}$ [7] of each cluster $i=\overline{1, N}$ are kept fixed to the values estimated from the data's intensity histogram; see Section 2.3

Here we formulate our model assuming WB MR data with two channels, but all the methods can be directly extended to the three and more channels case.

\subsection{Inter-station Intensity Calibration}

Inter-station data calibration is applied for equalization of the intensity of different stacks within the same scan. Depending on the anatomical region to which 
the acquired image stack corresponds, its intensity distribution can vary considerably from that of other stacks. The equalization is performed according to the approach described by Jäger and Hornegger [3]: each joint intensity histogram $H_{i}$ is registered to the histogram $H_{\text {ref }}$ of a reference station, and the calculated deformation fields are used for performing the intensity mapping. For performing all the registrations, we used the software tool "elastix" [8].

\subsection{Joint Multi-spectral Bias Correction via Possibilistic Clustering}

The CLIC framework estimates the bias field and the tissue probability maps based on two core assumptions: 1) The object consists of a finite number of tissue classes $N$, each of them having a constant true intensity value $c_{i}(i=\overline{1, N})$; and 2) The estimated bias field $b$ is slowly varying.

These assumptions allow to partition the entire image domain $\Omega$ into disjoint tissue regions $\Omega_{i}(i=\overline{1, N})$ via fuzzy classification 9 . The tissue classification and the bias field estimation is formulated as a single optimization problem. For the case of an additive bias field (1), this requires minimization of the following energy functional

$$
\mathcal{J}(U, \mathbf{c}, b) \triangleq \int \sum_{i=1}^{N} \int u_{i}^{q}(\mathbf{y}) K(\mathbf{x}-\mathbf{y})\left|I(\mathbf{y})-b(\mathbf{x})-c_{i}\right|^{2} d \mathbf{y} d \mathbf{x},
$$

where $c_{i}$ is the center of the cluster $i=\overline{1, N}$, and $u_{i}$ is the membership probability function of the corresponding tissue; $\mathbf{x}$ and $\mathbf{y}$ are Cartesian coordinates on $\Omega$; $q \geq 1$ is a scalar; and $K$ is truncated Gaussian kernel.

The energy (2) is convex with respect to the variables $b$, c and $U$, thus the model unknowns can easily be determined by differentiation with respect to the corresponding variables. For joint bias correction on two channels, the energy functional (2) for the vector image intensity $\mathbf{I}=\left(I_{1}, I_{2}\right)$, bias field $\mathbf{b}=\left(b_{1}, b_{2}\right)$, and centers $\mathbf{c}_{i}=\left(c_{1, i}, c_{2, i}\right)$ of each cluster $i=\overline{1, N}$ can be written as

$$
\mathcal{J}(U, \mathbf{c}, \mathbf{b})=\int \sum_{i=1}^{N} u_{i}^{q}(\mathbf{y}) d_{i}(\mathbf{y} ; \mathbf{c}, \mathbf{b}) d \mathbf{y},
$$

where

$$
\begin{gathered}
d_{i}(\mathbf{y} ; \mathbf{c}, \mathbf{b})=\int K(\mathbf{x}-\mathbf{y}) d \mathbf{x}\left[s_{11, i}\left(I_{1}(\mathbf{y})-b_{1}(\mathbf{x})-c_{1, i}\right)^{2}+\left(s_{12, i}+s_{21, i}\right) \times\right. \\
\left.\left(I_{1}(\mathbf{y})-b_{1}(\mathbf{x})-c_{1, i}\right)\left(I_{2}(\mathbf{y})-b_{2}(\mathbf{x})-c_{2, i}\right)+s_{22, i}\left(I_{2}(\mathbf{y})-b_{2}(\mathbf{x})-c_{2, i}\right)^{2}\right],
\end{gathered}
$$

and 6 ]

$$
\left(\begin{array}{ll}
s_{11, i} & s_{12, i} \\
s_{21, i} & s_{22, i}
\end{array}\right)=\left(\Sigma_{i}^{\mathrm{new}}\right)^{-1}\left|\Sigma_{i}^{\mathrm{new}}\right|^{1 / n_{d}} .
$$

Here we use an improved estimate $\Sigma_{i}^{\text {new }}$ [7] of the covariance matrix $\Sigma_{i}$, and $n_{d}$ is the data dimensionality (in our case $n_{d}=3$ ). 
Within the possibilistic clustering framework, the class membership functions are given by the following expression [6]

$$
\widehat{u}_{i}=\left[1+\left(\frac{d_{i}(\mathbf{I})}{\eta_{i}}\right)^{\frac{1}{q-1}}\right]^{-1}, \quad i=\overline{1, N},
$$

where $\eta_{i}$ is the bandwidth of each cluster. Our definition of this parameter is given in Section 2.3. Differentiation of (3) with respect to $\mathbf{b}$ gives us a following linear system of equations for calculating the bias field, whose solution can be written as

$$
\widehat{b}_{s, k}=T_{s}^{(3-k)} Q_{s}^{(k)}-T_{s}^{(3)} Q_{s}^{(3-k)}, \quad k=\overline{1,2},
$$

where the variables $P_{s}^{(1: 3)}, Q_{s}^{(1: 2)}$, and $T_{s}^{(1: 3)}$ are defined in the Appendix. The values of $u_{i}(i=\overline{1, N})$ and $\mathbf{b}$ are iteratively updated till convergence by using expressions given in equations (4) and (5).

\subsection{Algorithm Initialization}

Our algorithm is initialized by estimating the mean $c_{i}$, the covariance $\Sigma_{i}$, and the bandwidth $\eta_{i}=\int_{R_{i}} d_{i}(\mathbf{x}) d \mathbf{x}$ of each cluster $i=\overline{1, N}$ from segmented joint histogram. Based on the typical appearance of the joint histograms, we identify $N$ as the number of present clusters. Consequently, the joint histogram is automatically segmented using a region-growing-type algorithm into $N$ regions $R_{i}$ corresponding to each of the observed clusters.

\subsection{Constrained Optimization}

Overlap Constraint. Multi-station MR data is typically acquired with relatively large overlap between the neighboring stations. The real image intensities in the overlap region of each pair of neighboring stations should be approximately equal

$$
J(\mathbf{x})=J\left(D_{s}(\mathbf{x})\right), \quad \mathbf{x} \in O_{s}^{(2)}, \quad s=\overline{1, N_{s}-1} .
$$

This property can be used to further improve the quality of bias correction. Here index $s$ denotes the image stack; $N_{s}$ is the total number of stations in the data set; $O_{s}^{(1)}$ and $O_{s}^{(2)}$ are respectively the overlap regions of stack $S_{s}$ with the previous and the next stacks; and $D_{s}(\mathbf{x})$ is the function that maps $O_{s}^{(2)}$ onto $O_{s+1}^{(1)}$. Providing such extra knowledge into the system is especially important as the regions adjacent to the boundary are the most prone to geometric distortions.

Condition (6) is incorporated into our model as an optimization constraint

$$
\left.\mathcal{J}_{\text {constr }}^{\text {overlap }}=\mathcal{J}+\sum_{s=1}^{N_{s}-1} \boldsymbol{\lambda}_{s}\left[\left(K * \mathbf{I}_{s}-\mathbf{b}_{s}\right)-\left(K * \mathbf{I}_{s+1}-\mathbf{b}_{s+1}\right)\left(D_{s}\right)\right)\right],
$$

where $\boldsymbol{\lambda}_{s}=\left(\lambda_{s, 1}, \lambda_{s, 2}\right)\left(s=\overline{1, N_{s}-1}\right)$ is the vector of Lagrange multipliers [10] for both image channels for $\mathbf{x} \in O_{s}^{(2)}$. The full derivation of the equivalents of equations (5) for the constrained optimization case is presented in the Appendix 
Linearity Constraint. For the two-point Dixon imaging, each voxel belongs to one of the following classes: background (air), water, or fat. In order to better preserve the relation between the last two classes, we introduce the following constraint for $\mathbf{x} \in\left\{\mathbf{x}: \operatorname{argmax}_{i}\left(\mathbf{u}_{i}(\mathbf{x})\right) \in\left\{i_{\mathrm{w}}, i_{\mathrm{f}}\right\}\right\}:$

$$
\mathcal{J}_{\text {constr }}^{\text {linear }}=\mathcal{J}+\sum_{s=1}^{N_{s}} \lambda_{s} \sum_{k=1}^{2}(-1)^{3-k} a_{3-k}\left[\left(K * \mathbf{I}_{s, k}-\mathbf{b}_{s, k}\right)-c_{i_{\mathrm{w}}, k}\right] \text {, }
$$

where $\mathbf{a}=\mathbf{c}_{i_{\mathrm{w}}}-\mathbf{c}_{i_{\mathrm{f}}}$, and $i_{\mathrm{w}}$ and $i_{\mathrm{f}}$ denote correspondingly the background, the water, and the fat class. Such constraint imposes a linear relation between the two foreground classes (water and fat). Final expressions for the Lagrangian multiplier $\boldsymbol{\lambda}$ and the bias field $\mathbf{b}$ for the described constrained optimization problem are given in the Appendix.

\subsection{Intensity Standardization and Volume Reconstruction}

Inter-scan intensity standardization is applied when the analysis involves multiple different scans, e.g. for baseline-follow-up comparisons. In this case, WB volumes belonging to different patients or follow-up scans of the same patient require prior intensity calibration. We achieve this by registering joint histograms of the complete body volumes, using a similar approach to the one described in Section 2.1.

Finally, all the separately acquired image stacks are reconstructed into a single volume using the geometric information recorded by the scanner. Since the data is acquired with some overlap between the neighboring stations, the information from both stacks has to be combined in the overlap region. The combined image is constructed by using the image blending method [11.

\section{$3 \quad$ Experiments and Results}

In this section, we apply the described method to two large sets of WB MR data: multiple myeloma patients data set, and two-point water-fat Dixon data set. Bias correction performance of our method was validated by comparing it to that of the two state-of-the art methods: N4 [12] and the original CLIC algorithm [5].

MM Patients Data Set. The MM patients data set consisted of $24 \mathrm{WB}$ volumes of 8 patients, and was acquired on a commercial human WB 1.5T MR Philips Intera system. Each WB volume had both a $\mathrm{T}_{1}$-weighted $\left(\mathrm{T}_{1} \mathrm{~W}\right)$ and a Short Tau Inversion Recovery (STIR) or $\mathrm{T}_{2}$-STIR sequence. Number of scans per patient varied from 1 to 4 , with approximately six month interval between the followup scans. 3D image stacks with coronal slice direction were acquired with the overlap approximately equal to $5 \%$ of the total volume of the two stacks. For improved estimation of the bias field in the overlap area, the overlap optimization constraint introduced in Section 2.4 was used. Processing time on a typical volume of size $1500 \times 500 \times 50$ voxels was around 20 minutes. 


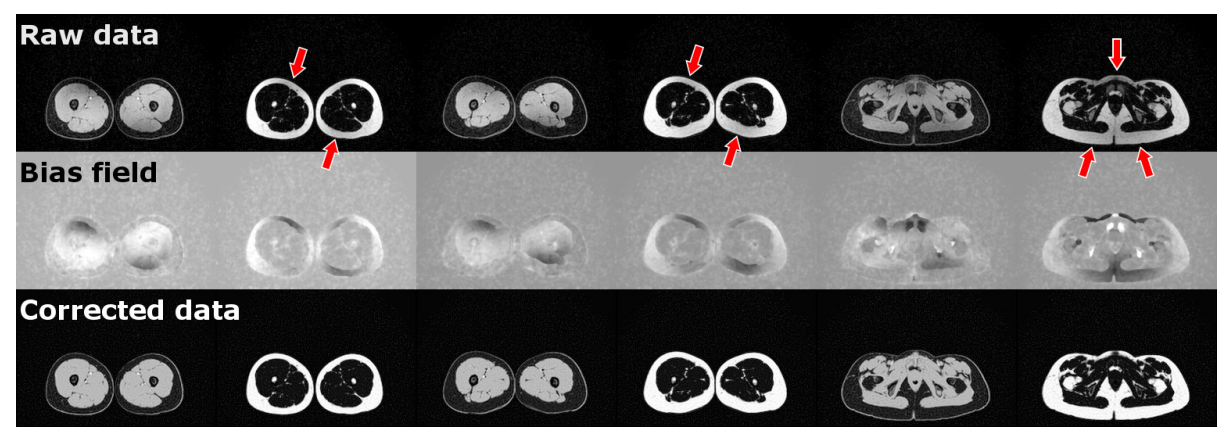

Fig. 1. Result of bias correction on a two-point Dixon data set. The areas of the most significant quality improvement are indicated by the arrows. The estimated inhomogeneity field is in nice agreement with characteristic $\mathrm{RF}$ wave propagation problems known in quadrature body coil transmission at 3T.

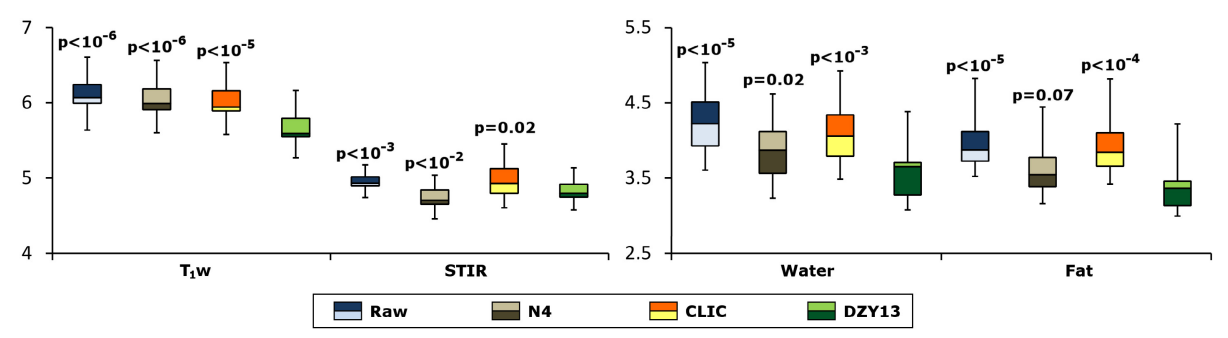

Fig. 2. Bias correction performance of our method on the MM patients data set (left) and the two-point Dixon data set (right). The histogram entropy of the raw data and that corrected by three methods: N4, CLIC, and the presented method (labeled as DZY13), is shown. Lower histogram entropy indicates better bias correction. Statistical significance was calculated using the paired Kolmogorov-Smirnov test with the DZY13 method as the reference.

Two-point Dixon Data Set. For two-point Dixon data, in vivo experiments were conducted on 8 healthy adults using a 3.0T clinical Philips Achieva Tx scanner. For simplicity and patient comfort reasons, the body coil was used for RF transmission and reception in the quadrature mode. 3D multi-station spoiled gradient echo (FFE) imaging was performed using a dual-echo-Dixon imaging sequence 13. with alternating readout gradients for water-fat separation. Bias correction on this data set was performed by using the algorithm described in Section 2, with the linearity optimization constraint. Processing time for both typical volume sizes $256 \times 256 \times 1520$ voxels and $336 \times 336 \times 960$ voxels was around 30 minutes.

A typical result of performing bias correction by our method is shown in Figure 1] The histogram entropy [4], which is widely applied for measuring the quality of bias correction when a ground truth is not available, was used for quantitative evaluation. The results with respect to the entropy intensity 
histogram of the raw images and the images corrected by our algorithm and two reference methods N4 and CLIC are shown in Figure 2, This analysis clearly indicates large quality improvement of the data corrected by our method, and its better performance in comparison with the reference state-of-the-art bias removal algorithms.

\section{Conclusions}

In this paper, we have presented a new algorithm for reconstruction of a complete volume from multiple separately acquired stations in multi-spectral WB MR data. Our method performs reconstruction of two types of intensity inhomogeneity, and combines all the acquired stations into a single volume. Among the novelties introduced in our algorithm compared to established methodology, the most important two are: 1) The joint processing (bias correction, calibration, standardization, etc.) on all available data channels; and 2) Integration of optimization constraints for improved estimation of the bias field. The described algorithm is applied to two different types of WB MR data, resulting in considerable quality improvement of the volumes reconstruction.

\section{References}

1. Kullberg, J., Angelhed, J.E., Lönn, L., Brandberg, J., Ahlström, H., Frimmel, H., Johansson, L.: Whole-body T1 mapping improves the definition of adipose tissue: Consequences for automated image analysis. J. Magn. Reson. Im. 24(2), 394-401 (2006)

2. Börnert, P., Aldefeld, B.: Principles of whole-body continuously-moving-table MRI. J. Magn. Reson. Im. 28(1), 1-12 (2008)

3. Jäger, F., Hornegger, J.: Nonrigid registration of joint histograms for intensity standardization in magnetic resonance imaging. IEEE Trans. Med. Imag. 28(1), 137-150 (2009)

4. Belaroussi, B., Milles, J., Carme, S., Zhu, Y.M., Benoit-Cattin, H.: Intensity nonuniformity correction in MRI: Existing methods and their validation. Med. Image Anal. 10(2), 234-246 (2006)

5. Li, C., Xu, C., Anderson, A.W., Gore, J.C.: MRI tissue classification and bias field estimation based on coherent local intensity clustering: A unified energy minimization framework. In: Prince, J.L., Pham, D.L., Myers, K.J. (eds.) IPMI 2009. LNCS, vol. 5636, pp. 288-299. Springer, Heidelberg (2009)

6. Krishnapuram, R., Keller, J.M.: A possibilistic approach to clustering. IEEE Trans. Fuz. Sys. 1(2), 98-110 (1993)

7. Babuška, R., van der Veen, P., Kaymak, U.: Improved covariance estimation for Gustafson-Kessel clustering. In: Proc. 2002 IEEE Int. Conf. Fuz. Sys (FUZZ-IEEE 2002), Honolulu, Hawaii, USA, vol. 2, pp. 1081-1085 (2002)

8. Klein, S., Staring, M., Murphy, K., Viergever, M., Pluim, J.: elastix: a toolbox for intensity-based medical image registration. IEEE Trans. Med. Imag. 29(1), 196-205 (2010)

9. Bezdek, J., Pal, S.: Fuzzy Models for Pattern Recognition, 1st edn. IEEE Press (1992) 
10. Bertsekas, D.P.: Constrained Optimization and Lagrange Multiplier Methods, 1st edn. Optimization and Neural Computation Series. Athena Scientific (1996)

11. Burt, P.J., Adelson, E.H.: A multiresolution spline with application to image mosaics. ACM Trans. Graph. 2, 217-236 (1983)

12. Tustison, N., Gee, J.: N4ITK: Nick's N3 ITK implementation for MRI bias field correction. Insight J. (January-June 2009)

13. Eggers, H., Brendel, B., Duijndam, A., Herigault, G.: Dual-echo Dixon imaging with flexible choice of echo times. Magn. Reson. Med. 65(1), 96-107 (2011)

\section{Appendix}

We introduce the following notation for $k=\overline{1,2}$ and $l=\overline{1,3}$

$$
\begin{aligned}
& P_{s}^{(k)}=2 K * \sum_{i=1}^{N} u_{i, s}^{q} s_{k k, i}, \quad P_{s}^{(3)}=K * \sum_{i=1}^{N} u_{i, s}^{q}\left(s_{12, i}+s_{21, i}\right), \\
& Q_{s}^{(k)}=K * \sum_{i=1}^{N} u_{i, s}^{q}\left[2\left(I_{s, k}-\mathbf{c}_{i, k}\right) s_{k k, i}+\left(I_{s, 3-k}-\mathbf{c}_{i, 3-k}\right)\left(s_{12, i}+s_{21, i}\right)\right], \\
& T_{s}^{(l)}=\frac{P_{s}^{(l)}}{P_{s}^{(1)} P_{s}^{(2)}-\left(P_{s}^{(3)}\right)^{2}}, \quad R_{s}^{(k)}=K * I_{s, k}-T_{s}^{(3-k)} Q_{s}^{(k)}+T_{s}^{(3)} Q_{s}^{(3-k)},
\end{aligned}
$$

Using the optimization constraint (7), the following equivalent of the expression (5) can be derived for $k=\overline{1,2}$

$$
\widehat{b}_{s, k}^{(\mathrm{c})}=\widehat{b}_{s, k}+T_{s}^{(3)}\left(\lambda_{s-1,3-k}\left(D_{s}^{-1}\right)+\lambda_{s, 3-k}\right)-T_{s}^{(3-k)}\left(\lambda_{s-1, k}\left(D_{s}^{-1}\right)+\lambda_{s, k}\right),
$$

where $D_{s}^{-1}(\mathbf{x})$ denotes the inverse mapping of $O_{s+1}^{(1)}$ onto $O_{s}^{(2)}$.

Combining (9) with the constraint (7), we obtain a linear system of equations for calculating the Lagrange multipliers $\boldsymbol{\lambda}$. Its solution for $k=\overline{1,2}$ is given by

$$
\lambda_{s, k}=\frac{V_{s}^{(k)} W_{s}^{(k)}-V_{s}^{(3)} W_{s}^{(3-k)}}{V_{s}^{(1)} V_{s}^{(2)}-\left(V_{s}^{(3)}\right)^{2}}, V_{s}^{(l)}=T_{s}^{(l)}+T_{s+1}^{(l)}\left(D_{s}\right), W_{s}^{(k)}=R_{s}^{(k)}-R_{s+1}^{(k)}\left(D_{s}\right) .
$$

Substituting this solution into (9) gives us the resulting expressions for $\mathbf{b}$.

For the case of the optimization constraint (8), the following expression for the bias field $\widehat{\mathbf{b}}^{(\mathrm{c})}$ is obtained

$$
\widehat{b}_{s, k}^{(\mathrm{c})}=\widehat{b}_{s, k}+(-1)^{3-k} \lambda_{s}\left(a_{3-k} T_{s}^{(3-k)}+a_{k} T_{s}^{(3)}\right), \quad k=\overline{1,2} .
$$

Substituting the latter into the constraint (8) gives us

$$
\lambda_{s, k}=\frac{a_{2}\left(K * I_{s, 1}-\widehat{b}_{s, 1}-c_{1, i_{\mathrm{w}}}\right)-a_{1}\left(K * I_{s, 2}-\widehat{b}_{s, 2}-c_{2, i_{\mathrm{w}}}\right)}{a_{1}^{2} T_{s}^{(1)}+a_{2}^{2} T_{s}^{(2)}+2 a_{1} a_{2} T_{s}^{(3)}}, \quad k=\overline{1,2} .
$$

This solution, together with (10), gives us the final expression for the bias $\mathbf{b}$. 